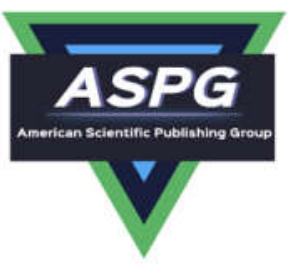

\title{
Neutrosophic Event-Based Queueing Model
}

\author{
Mohamed Bisher Zeina \\ Dept. of Mathematical Statistics, Faculty of Sciences, University of Aleppo, Aleppo, Syria \\ bisherzeina@alepuniv.edu.sy
}

\begin{abstract}
In this paper we have defined the concept of neutrosophic queueing systems and defined its neutrosophic performance measures. An important application of neutrosophic logic in queueing systems we face in real life were discussed, that is the neutrosophic events accuring times, because of its wide applications in networking and simulating communication systems specialy when probability distribution is not known, and because it's more realistic to consider and to not ignore the imprecise events times. Event-based table of a neutrosophic queueing system was presented and its neutrosophic performance measures were derived, i.e. neutrosophic mean waiting time in queue, neutrosophic mean waiting time in system, neutrosophic expected number of customers in queue and neutrosophic expected number of customers in system. Neutrosophic Little's Formulas (NLF) were also defined which is a main tool in queueing systems problems to make it easier finding performance measures from each other.
\end{abstract}

Keywords: Neutrosophic Logic; Queueing Theory; Performance Measures; Little's Formula

\section{Introduction}

Neutrosophic Logic that was presented by F.Smarandache in 1995 as generalization of intuitionistic fuzzy logic has lots of applications in many branches of science specially in probability, statistics and operation research $[1,2,3,4,5,6,7]$. In these three mentioned branches of science we face lots of imprecise, ambiguity, fluctuation and incomplete data $[3,4,5,6,7]$. Before the foundation of neutrosophic logic we couldn't deal with these cases $[8,9,10,11,12]$. Queueing theory or waiting lines theory is one of the most important applications of probability theory and operations research presented by Erlang in 1909, which is a modelling of systems where customers have to wait unitl they get required services [13]. Queueing theory has many applications in traffic flow, scheduling, designing communication networks and facility design $[13,14,15]$.

In queueing theory, efficiency of service providing systems can be determined depending on the time that customer must wait unitl he takes his desired service and the cost that is resulting from system's design $[14,15]$.

Modeling a queuenig system depends on lots of characteristics including: interarrival times, departures times, servicing times, number of servers, etc. but these are not always determined exactly and most times are inaccurate $[3,10,14]$.

In classical cirsp qeues researcher often use rates, midpoints or most probable values to represent in queue's parameters $[16,17]$. The extension of classical queueing theory to neutrosophic queueing theory means that the elements of the queue are imprecise, i.e. times of arrivals, timer of taking services and departures are not known exactly. In this paper, the definition of neutrosophic performance measures of a general queue depending on event- 
based table and NLF was introduced, then a solved example was presented to show the power of neutrosophic crisp sets $[3,5,18]$ in solving problems better and more accurate than in classical crisp logic.

\section{Neutrosophic queues}

\subsection{Definitions and notions}

Suppose that the queueing system consists of one waiting line. Arriving customer takes his service from only one server. If the server is busy then arriving customer waits in the queue until the server is empty. The server can serve one customer at time according to first comes first served policy. The customer departs after he takes his service.

Let by definition:

- $\quad N A^{(n)}$ denotes the neutrosophic time of customer (n) arrival.

- $N S^{(n)}$ denotes the neutrosophic service time of customer (n).

- $N T^{(n)}$ denotes the neutrosophic interarrival time of customer (n) and customer (n+1) so:

$$
N T^{(n)}=N A^{(n+1)}-N A^{(n)}
$$

- $N U^{(n)}$ denotes the neutrosophic time that customer (n) starts taking the service so:

$$
N U^{(n+1)}=\max \left(N D^{(n)}, N A^{(n+1)}\right)
$$

- $N D^{(n)}$ denotes the neutrosophic time of customer (n) departure that is:

- $\quad N W_{q}^{(n)}$ denotes waiting time in queue of customer (n) so:

$$
N D^{(n)}=N U^{(n)}+N S^{(n)}
$$

$$
N W_{q}^{(n)}=N U^{(n)}-N A^{(n)}
$$

- $\quad N W^{(n)}$ denotes waiting time in system of customer (n) so:

$$
N W^{(n)}=N W_{q}^{(n)}+N S^{(n)}
$$

- $\quad N W_{q}$ denotes the neutrosophic mean waiting time in queue.

- $\quad N W$ denotes the neutrosophic mean waiting time in system.

- $N L_{q}$ denotes the neutrosophic expected number of customers in queue.

- $\quad N L$ denotes the neutrosophic expected number of customers in system.

NLF will be then (based on classical little's formula in $[14,15]$ ):

$$
\begin{gathered}
N L=\lambda * N W \\
N L_{q}=\lambda * N W_{q}
\end{gathered}
$$

Where $\lambda$ is the arrival rate, that is total number of arrivals divided by the time between last departure and starting time.

Among this paper, neutrosophic statistical numbers presented in [19] will be used, which is an effective method to describe indeterminacy in data specially data that it is not useful to be presented in the form (T, I, F) like parameters of distributions, statistical data, statistical data tables, frequency tables, time series, etc $[4,5,10,19,20]$.

\subsection{Example}

Suppose that we have the following event-based table of a queueing system representing arriving customers to an $\operatorname{ATM}$ (all times are in minutes):

\begin{tabular}{|c|c|c|}
\hline Customer & $\boldsymbol{N A ^ { ( n ) }}$ & $\boldsymbol{N} \boldsymbol{S}^{(\boldsymbol{n})}$ \\
\hline 1 & 0 & {$[1,2]$} \\
\hline 2 & {$[3,4]$} & 4 \\
\hline
\end{tabular}




\begin{tabular}{|c|c|c|}
\hline 3 & 6 & 2 \\
\hline 4 & 8 & 1 \\
\hline 5 & 12 & {$[1,2]$} \\
\hline 6 & {$[13,14]$} & 2 \\
\hline
\end{tabular}

Table 1: Input data

Lets calculate the performance measures of this ATM system according to Neutrosophic queues then according to cirsp queues:

\section{Neutrosophic Solution:}

For customer 1: we start when the system is empty so $N A^{(1)}=0$ that is the arrival time of ther first customer.

Since the system is empty then the arrival customer will be served without any waiting so $N U^{(1)}=0$.

The service time for this customer is $N S^{(1)}=[1,2]$ so the departure time will be $N D^{(1)}=N U^{(1)}+N S^{(1)}=0+$ $[1,2]=[1,2]$ that is the time of start serving the customer plus the serving time.

The waiting time in queue is the difference between starting serving the customer $N U^{(1)}$ and arriving time of this customer $N A^{(1)}$ so : $N W_{q}^{(1)}=N U^{(1)}-N A^{(1)}=0-0=0$.

Waiting time in system is sum of waiting time in queue and serving time that is : $N W^{(1)}=N W_{q}^{(1)}+N S^{(1)}=0+$ $[1,2]=[1,2]$.

Interarrival time between first and next customer is $N T^{(1)}=N A^{(2)}-N A^{(1)}=[3,4]-0=[3,4]$.

For customer 2: $N A^{(2)}=[3,4]$ that is the arrival time of the second customer.

The service starting time of this customer (customer 2) will be its arriving time if the server is empty or it will be immediately after the departure time of the previous customer if the server is busy, so it's $N U^{(2)}=$ $\max \left(N D^{(1)}, N A^{(2)}\right)=\max ([1,2],[3,4])=[3,4]$.

The service time for this customer is $N S^{(2)}=4$ so the departure time will be $D^{(2)}=N U^{(2)}+N S^{(2)}=[3,4]+4=$ $[7,8]$.

The waiting time in queue is the difference between starting serving the customer $N U^{(2)}$ and arriving time of this customer $N A^{(2)}$ so : $N W_{q}^{(2)}=N U^{(2)}-N A^{(2)}=[3,4]-[3,4]=0$.

Waiting time in system is sum of waiting time in queue and serving time that is : $N W^{(2)}=N W_{q}^{(2)}+N S^{(2)}=0+$ $4=4$.

Interarrival time between second and third customer is $N T^{(2)}=N A^{(3)}-N A^{(2)}=6-[3,4]=[2,3]$. 
For customer 3: $N A^{(3)}=6$.

The service starting time of this customer will be $N U^{(3)}=\max \left(N D^{(2)}, N A^{(3)}\right)=\max ([7,8], 6)=[7,8]$.

The service time for this customer is $N S^{(3)}=2$ so the departure time will be $D^{(3)}=N U^{(3)}+N S^{(3)}=[7,8]+2=$ $[9,10]$.

The waiting time in queue is: $N W_{q}^{(3)}=N U^{(3)}-N A^{(3)}=[7,8]-6=[1,2]$.

Waiting time in system is : $N W^{(3)}=N W_{q}^{(3)}+N S^{(3)}=[1,2]+2=[3,4]$.

Interarrival time between third and forth customer is $N T^{(3)}=N A^{(4)}-N A^{(3)}=8-6=2$.

And so on.. We can form the following table:

\begin{tabular}{|c|c|c|c|c|c|c|c|}
\hline Customer & $\boldsymbol{N} \boldsymbol{A}^{(\boldsymbol{n})}$ & $\boldsymbol{N} \boldsymbol{S}^{(\boldsymbol{n})}$ & $\boldsymbol{N} \boldsymbol{T}^{(\boldsymbol{n})}$ & $\boldsymbol{N} \boldsymbol{U}^{(\boldsymbol{n})}$ & $\boldsymbol{N} \boldsymbol{D}^{(\boldsymbol{n})}$ & $\boldsymbol{N} \boldsymbol{W}_{\boldsymbol{q}}^{(\boldsymbol{n})}$ & $\boldsymbol{N} \boldsymbol{W}^{(\boldsymbol{n})}$ \\
\hline 1 & 0 & {$[1,2]$} & {$[3,4]$} & 0 & {$[1,2]$} & 0 & {$[1,2]$} \\
\hline 2 & {$[3,4]$} & 4 & {$[2,3]$} & {$[3,4]$} & {$[7,8]$} & 0 & 4 \\
\hline 3 & 6 & 2 & 2 & {$[7,8]$} & {$[9,10]$} & {$[1,2]$} & {$[3,4]$} \\
\hline 4 & 8 & 1 & 4 & {$[9,10]$} & {$[10,11]$} & {$[1,2]$} & {$[2,3]$} \\
\hline 5 & 12 & {$[1,2]$} & {$[1,2]$} & 12 & {$[13,14]$} & 0 & {$[1,2]$} \\
\hline 6 & {$[13,14]$} & 2 & - & {$[13,14]$} & {$[15,16]$} & 0 & 2 \\
\hline
\end{tabular}

Table 2: Neutrosophic event-based queue table

Now we can calculate the performance measures as follows:

$N W_{q}=\frac{\sum_{k=1}^{6}\left[N W_{q}^{(n)}\right]_{k}}{6}=\frac{0+0+[1,2]+[1,2]+0+0}{6}=\frac{[2,4]}{6}=[0.33,0.67]$ minutes

Which means that customer will wait in the queue between 0.33 and 0.67 mins or between 20 and 40 secs before starting being served.

$N W=\frac{\sum_{k=1}^{6}\left[N W^{(n)}\right]_{k}}{6}=\frac{[1,2]+4+[3,4]+[2,3]+[1,2]+2}{6}=\frac{[13,17]}{6}=[2.17,2.833]$ minutes

Which means that customer will stay in the system between 2.17 and 2.833 that is between 2 mins and 10 secs and 2 mins and 50 secs from the time he arrives until he departs from the system.

We have also $\lambda=\frac{6}{16}=0.375$ that is the arrival rate because we totally have 6 customers arrived during 16 minutes. 
Now using Little's formula we have:

$N L=\lambda * N W=0.375 *[2.17,2.833]=[0.81375,1.062375]$ customers

That means expected number of customers in the system will be between 0.81375 and 1.062375 customers.

$N L_{q}=\lambda * N W_{q}=0.375 *[0.33,0.67]=[0.12375,0.25125]$ customers

That means expected number of custoemrs in queue will range between 0.12375 and 0.25125 customers.

\section{Crisp Solution:}

To solve this kind of problems using crisp queues we may take midpoints of intervals, so:

\begin{tabular}{|c|c|c|}
\hline Customer & $\boldsymbol{A}^{(\boldsymbol{n})}$ & $\boldsymbol{S}^{(\boldsymbol{n})}$ \\
\hline 1 & 0 & 1.5 \\
\hline 2 & 3.5 & 4 \\
\hline 3 & 6 & 2 \\
\hline 4 & 8 & 1 \\
\hline 5 & 12 & 1.5 \\
\hline 6 & 13.5 & 2 \\
\hline
\end{tabular}

Table 3: Input data converted into crisp data

For customer 1: Since the system is empty then the arrival customer will be served without any waiting so $U^{(1)}=0$.

The service time for this customer is approximatley $S^{(1)}=1.5$ so the departure time will be approximately $D^{(1)}=$ $U^{(1)}+S^{(1)}=0+1.5=1.5$ that is the apprpximated time of start serving the customer plus the approximated serving time.

The waiting time in queue is the difference between starting serving the customer $U^{(1)}$ and arriving time of this customer $A^{(1)}$ so : $W_{q}^{(1)}=U^{(1)}-A^{(1)}=0-0=0$.

Waiting time in system is sum of waiting time in queue and serving time that is : $W^{(1)}=W_{q}^{(1)}+S^{(1)}=0+1.5=$ 1.5 .

Interarrival time between first and next customer is approximately $T^{(1)}=A^{(2)}-A^{(1)}=3.5-0=3.5$

For customer 2: $A^{(2)}=3.5$ that is the approximated arrival time of the second customer. 
The service starting time of this customer will be its arriving time if the server is empty or it will be immediately after the departure time of the previous customer if the server is busy, so it's $U^{(2)}=\max \left(D^{(1)}, A^{(2)}\right)=$ $\max (1.5,3.5)=3.5$

The service time for this customer is $S^{(2)}=4$ so the departure time will be $D^{(2)}=U^{(2)}+S^{(2)}=3.5+4=7.5$.

The waiting time in queue is the difference between starting serving the customer $U^{(2)}$ and arriving time of this customer $A^{(2)}$ so : $W_{q}^{(2)}=U^{(2)}-A^{(2)}=3.5-3.5=0$.

Waiting time in system is sum of waiting time in queue and serving time that is : $W^{(2)}=W_{q}^{(2)}+S^{(2)}=0+4=4$.

Interarrival time between second and third customer is $T^{(2)}=A^{(3)}-A^{(2)}=6-3.5=2.5$

And so on.. We can form the following table:

\begin{tabular}{|c|c|c|c|c|c|c|c|}
\hline Customer & $\boldsymbol{A}^{(\boldsymbol{n})}$ & $\boldsymbol{S}^{(\boldsymbol{n})}$ & $\boldsymbol{T}^{(\boldsymbol{n})}$ & $\boldsymbol{U}^{(\boldsymbol{n})}$ & $\boldsymbol{D}^{(\boldsymbol{n})}$ & $\boldsymbol{W}_{\boldsymbol{q}}^{(\boldsymbol{n})}$ & $\boldsymbol{W}^{(\boldsymbol{n})}$ \\
\hline 1 & 0 & 1.5 & 3.5 & 0 & 1.5 & 0 & 1.5 \\
\hline 2 & 3.5 & 4 & 2.5 & 3.5 & 7.5 & 0 & 4 \\
\hline 3 & 6 & 2 & 2 & 7.5 & 9.5 & 1.5 & 3.5 \\
\hline 4 & 8 & 1 & 4 & 9.5 & 10.5 & 1.5 & 2.5 \\
\hline 5 & 12 & 1.5 & 1.5 & 12 & 13.5 & 0 & 1.5 \\
\hline 6 & 13.5 & 2 & - & 13.5 & 15.5 & 0 & 2 \\
\hline
\end{tabular}

Table 4: Crisp event-based queue table

Now performance measures can be calculated as follows:

$W_{q}=\frac{\sum_{k=1}^{6}\left[W_{q}^{(n)}\right]_{k}}{6}=\frac{0+0+1.5+1.5+0+0}{6}=\frac{3}{6}=0.5$ minutes $\in[0.33,0.67]=N W_{q}$

$W=\frac{\sum_{k=1}^{6}\left[W^{(n)}\right]_{k}}{6}=\frac{1.5+4+3.5+2.5+1.5+2}{6}=\frac{15}{6}=2.5$ minutes $\in[2.17,2.833]=N W$

We had before $\lambda=0.375$, now using crisp Little's formula:

$L=\lambda * W=0.375 * 2.5=0.9375$ customers $\in[0.81375,1.062375]=N L$

$L_{q}=\lambda * W_{q}=0.375 * 0.5=0.1875$ customers $\in[0.12375,0.25125]=N L_{q}$ 
We notice that neutrosophic solutions are more accurate and realistic than crisp solutions.

\section{Conclusions}

In this article, we discussed basics of neutrosophic queueing theory and have shown the power of neutrosophic logic in modelling queues with imprecise and incomplete inputs in even-based tables of queues. We solved an example which contains indetermined times of arrivals, departures, services and services starting times and calculated the neutrosophic mean waiting time in queue, neutrosophic mean waiting time in system, neutrosophic expected number of customers in queue and neutrosophic expected number of customers in system then compared it to crisp solutions.

We found that neutrosophic solutions can be considered as an extension to crisp solutions, also neutrosophic solutions are more accurate than crisp solutions.

In future work author looking forward to study more complex applications of neutrosophic logic to more genereal queueing problems like bulk queues, balk queues, networks of queues and impatient customers behaviour in queues like jockeying and reneging.

Acknowledgment: "The author is highly thankful to the Editor-in-Chief and the reviewers for their valuable comments and suggestions that improve the quality of paper".

Funding: "This research received no external funding"

Conflicts of Interest: "The authors declare no conflict of interest."

\section{References}

[1] Smarandache.F., "Introduction to Neutrosophic Measure, Neutrosophic Integral, and Neutrosophic Probability", Sitech-Education Publisher, Craiova - Columbus, 2013

[2] Atanassov .k, "Intuitionistic fuzzy sets," In V. Sgurev, ed., ITKRS Session, Sofia, June 1983, Central Sci. and Techn. Library, Bulg. Academy of Sciences, 1984.

[3] Salama, A. A. and Smarandache, F. "Neutrosophic crisp probability theory \& decision making process." Critical Review: A Publication of Society for Mathematics of Uncertainty, vol. 12, p. 34-48, 2016.

[4] Alhabib, R., A. A. Salama, "Using Moving Averages To Pave The Neutrosophic Time Series", International Journal of Neutrosophic Science, Vol. 3, 2020

[5] Alhabib, R., Ranna, M., Farah, H. and Salama, A. " Some neutrosophic probability distributions," Neutrosophic Sets and Systems, 22, pp.30-38, 2018.

[6] Sapan Kumar Das; S.A. Edalatpanah, "A new ranking function of triangular neutrosophic number and its application in integer programming", International Journal of Neutrosophic Science, Vol. 4, No. 2, 02020

[7] Christianto.V. ,Boyd. R.N. , Smarandach.F. , "Three possible applications of Neutrosophic Logic in Fundamental and Applied Sciences," International Journal of Neutrosophic Science, Volume 1, Issue 2, PP: 90-95, 2020.

[8] Smarandache. F, "Neutrosophic set a generalization of the intuitionistic fuzzy sets", Inter. J. Pure Appl. Math., 24, 287 - 297, 2005

[9] Smarandache, F., “A Unifying Field in Logics: Neutrosophic Logic. Neutrosophy, Neutrosophic Set, Neutrosophic Probability”, American Research Press, Rehoboth, NM, 1999

[10] Smarandache, F.” Introduction to Neutrosophic statistics," Sitech \& Education Publishing, 2014

[11] Hatip.A., "The Special Neutrosophic Functions" , International Journal of Neutrosophic Science,Vol. 4, 2020 
[12] A. Chakraborty, A "New Score Function of Pentagonal Neutrosophic Number and its Application in Networking Problem”, International Journal of Neutrosophic Science, Volume 1, Issue 1, pp. 40-51, 2020

[13] Erlang, Agner Krarup, "The theory of probabilities and telephone conversations" . NYT Tidsskrift for Matematik B. 20: 33-39, 1909.

[14] F Shortle J.; M Thompson J.; Gross D.; M Harris C., "Fundamentals of Queueing Theory", 5th ed.; Wiley: United States of America, 2018

[15] Nick T. Thomopoulos; "Fundamentals of Queuing Systems", 1st ed.; Springer: United States of America, 2012

[16] Balter. M.H., "Performance Modeling and Design of Computer Systems: Queueing Theory in Action", Cambridge, 2013

[17] Robertazzi T.G., "Computer Networks and Systems: Queueing Theory and Performance Evaluation”, $2^{\text {nd }}$ Edition, Springer, 1994

[18] Salama.A.A, Smarandache. F, Neutrosophic Crisp Set Theory. Education Publishing, Columbus, 2015.

[19] Patro.S.K, Smarandache. F., "The Neutrosophic Statistical Distribution, More Problems, More Solutions", Neutrosophic Sets and Systems, Vol. 12, 2016

[20] Kawther Fawzi Hamza Alhasan, F. Smarandache, "Neutrosophic Weibull distribution and Neutrosophic Family Weibull Distribution", Neutrosophic Sets and Systems, vol. 28, pp. 191-199, 2019 